\title{
Analysis of buckling phenomenon of rectangular laminated plates with a hole under different boundary conditions
}

\author{
Ahmed El Bouhmidi ${ }^{1}$, Mohamed Rougui ${ }^{1}$ \\ ${ }^{1}$ Mohammed V University in Rabat, Department GUE, LGCE High School of Technology- Salé CEDoc-EMI, Morocco
}

\begin{abstract}
In this research, buckling behavior of perforated rectangular plate of symmetric and anti-symmetric laminated composite is investigated based on Finite element analysis. The presence of hole may cause redistribution of stresses in plates with reduction of stability. The aim of the current paper is to find critical buckling load by using the (FSDT) first-order shear deformation theory in concomitance with the variational energy method. The load depends on many factors like as diameter of circular hole, different boundary conditions, lay-up sequences, length/thickness ratio and angle of ply orientation. The result is shown in graphical forms for various boundary conditions.
\end{abstract}

\section{Introduction}

Laminated composite plates are widely used in many engineering structures including automotive bridges wind-turbine blades and aviation, due to their lower weights compared to metal structures. Frequently, such plates contain holes of various shapes, for example for access or for weight savings and these perforation or openings often require some type of reinforcing structure to control local structural deformations and stresses near the cutout. In addition, these structures may experience compression loads during operation, and thus their buckling response characteristics must be understood and accurately predicted in order to determine effective designs and safe operating conditions for these structures. Thus in the design context buckling analysis plays a crucial role [4].

Hence, the analytical buckling solutions are inaccessible, and various numerical methods need to be developed for analyzing such plates. In the literature, few published studies investigated the buckling of laminated composite plates with a cutout $[4,7]$.

This paper deals with buckling of symmetrically and anti-symmetrically laminated composite plates with circular hole under uniaxial static loadings "figure 1". The FSDT plate deformation theory and the variational energy method are employed in mathematical formulation, and the finite element technique is used for finding critical loads. The effects on critical load by hole size, plate thickness ratio, material modulus ratio, plylamination geometry, loading types, and boundary conditions are investigated.

\section{Theoretical background}

Consider a symmetrically or anti-symmetrically laminated rectangular plate of length $a$, width $b$, central circular hole of diameter $d$ and thickness $t$ which consists of six orthotropic laminas [12].

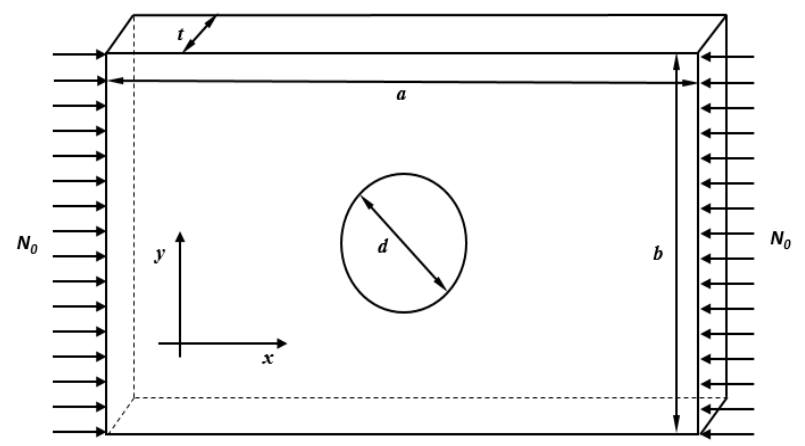

Fig. 1. Geometry of rectangular plate.

In this study, first-order shear deformation theory (FSDT) is employed to analyse the problem and the following displacement field is assumed $[8,10]$

$$
\left\{\begin{array}{c}
u(x, y, z)=u_{0}(x, y)+z \phi_{x}(x, y) \\
v(x, y, z)=v_{0}(x, y)+z \phi_{y}(x, y) \\
w(x, y, z)=w_{0}(x, y)
\end{array}\right.
$$

where $\left(u_{0}, v_{0}, w_{0}\right)$ denote the displacements of a point on the plane $z=0$, and

$$
\frac{\partial u}{\partial z}=\phi_{x}, \frac{\partial v}{\partial z}=\phi_{y}
$$


which indicate that $\phi_{x}$ and $\phi_{y}$ are the rotations of a transverse normal about the $y$ - and $x$ - axes, respectively.

The strains associated with the displacement field (1) are given by:

$$
\varepsilon_{B}=\left\{\begin{array}{l}
\varepsilon_{x x} \\
\varepsilon_{y y} \\
\gamma_{x y}
\end{array}\right\}=\left\{\begin{array}{c}
\frac{\partial u_{0}}{\partial x} \\
\frac{\partial v_{0}}{\partial y} \\
\frac{\partial u_{0}}{\partial y}+\frac{\partial v_{0}}{\partial x}
\end{array}\right\}+z\left\{\begin{array}{c}
\frac{\partial \phi_{x}}{\partial x} \\
\frac{\partial \phi_{y}}{\partial y} \\
\frac{\partial \phi_{x}}{\partial y}+\frac{\partial \phi_{y}}{\partial x}
\end{array}\right\}
$$

where $\varepsilon_{B}$ is the bending part

$$
\varepsilon_{S}=\left\{\begin{array}{l}
\gamma_{y z} \\
\gamma_{x z}
\end{array}\right\}=\left\{\begin{array}{l}
\phi_{y}+\frac{\partial w_{0}}{\partial y} \\
\phi_{x}+\frac{\partial w_{0}}{\partial x}
\end{array}\right\}
$$

and $\varepsilon_{S}$ is the transverse shear part.

The resultant force is obtained by integrating the stresses through the thickness of laminas [1,9]:

$$
\begin{aligned}
\left\{\begin{array}{l}
N_{x x} \\
N_{y y} \\
N_{x y}
\end{array}\right\} & =\int_{\frac{-t}{2}}^{\frac{t}{2}}\left\{\begin{array}{l}
\sigma_{x x} \\
\sigma_{y y} \\
\tau_{x y}
\end{array}\right\} d z=\sum_{i=1}^{n} \int_{t_{i-1}}^{t_{i}}\left\{\begin{array}{c}
\sigma_{x x} \\
\sigma_{y y} \\
\tau_{x y}
\end{array}\right\} d z \\
& =\left(\begin{array}{lll}
A_{11} & A_{12} & A_{16} \\
A_{12} & A_{22} & A_{26} \\
A_{16} & A_{26} & A_{66}
\end{array}\right)\left\{\begin{array}{c}
\frac{\partial u_{0}}{\partial x} \\
\frac{\partial v_{0}}{\partial y} \\
\frac{\partial u_{0}}{\partial y}+\frac{\partial v_{0}}{\partial x}
\end{array}\right\} \\
& +\left(\begin{array}{lll}
B_{11} & B_{12} & B_{16} \\
B_{12} & B_{22} & B_{26} \\
B_{16} & B_{26} & B_{66}
\end{array}\right)\left\{\begin{array}{c}
\frac{\partial \phi_{x}}{\partial x} \\
\frac{\partial \phi_{y}}{\partial y} \\
\frac{\partial \phi_{x}}{\partial y}+\frac{\partial \phi_{y}}{\partial x}
\end{array}\right\}
\end{aligned}
$$

and we obtained the resultant moment with the same way:

$$
\begin{aligned}
\left\{\begin{array}{l}
M_{x x} \\
M_{y y} \\
M_{x y}
\end{array}\right\} & =\int_{\frac{-t}{2}}^{\frac{t}{2}}\left\{\begin{array}{l}
\sigma_{x x} \\
\sigma_{y y} \\
\tau_{x y}
\end{array}\right\} z d z=\sum_{i=1}^{n} \int_{t_{i-1}}^{t_{i}}\left\{\begin{array}{l}
\sigma_{x x} \\
\sigma_{y y} \\
\tau_{x y}
\end{array}\right\} z d z \\
& =\left(\begin{array}{lll}
B_{11} & B_{12} & B_{16} \\
B_{12} & B_{22} & B_{26} \\
B_{16} & B_{26} & B_{66}
\end{array}\right)\left\{\begin{array}{c}
\frac{\partial u_{0}}{\partial x} \\
\frac{\partial v_{0}}{\partial y} \\
\frac{\partial u_{0}}{\partial y}+\frac{\partial v_{0}}{\partial x}
\end{array}\right\}+\left(\begin{array}{lll}
D_{11} & D_{12} & D_{16} \\
D_{12} & D_{22} & D_{26} \\
D_{16} & D_{26} & D_{66}
\end{array}\right)\left\{\begin{array}{c}
\frac{\partial \phi_{x}}{\partial x} \\
\frac{\partial \phi_{y}}{\partial y} \\
\frac{\partial \phi_{x}}{\partial y}+\frac{\partial \phi_{y}}{\partial x}
\end{array}\right\}
\end{aligned}
$$

Equations relating the shear force to the shear strains can be computed as:

$$
\begin{aligned}
\left\{\begin{array}{l}
R_{y z} \\
R_{x z}
\end{array}\right\} & =k_{S} \int_{\frac{-t}{2}}^{\frac{t}{2}}\left\{\begin{array}{l}
\tau_{y z} \\
\tau_{x z}
\end{array}\right\}=k_{S} \sum_{i=1}^{n} \int_{t_{i-1}}^{t_{i}}\left\{\begin{array}{l}
\tau_{y z} \\
\tau_{x z}
\end{array}\right\}_{i} d z \\
& =k_{S}\left(\begin{array}{ll}
A_{44} & A_{45} \\
A_{45} & A_{55}
\end{array}\right)\left\{\begin{array}{l}
\phi_{y}+\frac{\partial w_{0}}{\partial y} \\
\phi_{x}+\frac{\partial w_{0}}{\partial x}
\end{array}\right\}
\end{aligned}
$$

where $k_{S}(=5 / 6)$ is the shear correction factor [5-6].

Equilibrium equations can be written in terms of the displacement field as follows [10]:

$$
\begin{aligned}
& D_{11} \frac{\partial^{2} \phi_{x}}{\partial x^{2}}+\left(D_{12}+D_{66}\right) \frac{\partial^{2} \phi_{y}}{\partial x \partial y}+D_{66} \frac{\partial^{2} \phi_{x}}{\partial y^{2}}-A_{55} k_{S}\left(\phi_{x}+\frac{\partial w}{\partial x}\right)=0 \\
& D_{22} \frac{\partial^{2} \phi_{y}}{\partial y^{2}}+\left(D_{12}+D_{66}\right) \frac{\partial^{2} \phi_{x}}{\partial x \partial y}+D_{66} \frac{\partial^{2} \phi_{y}}{\partial x^{2}}-A_{44} k_{S}\left(\phi_{y}+\frac{\partial w}{\partial y}\right)=0 \\
& A_{55} k_{S}\left(\frac{\partial \phi_{x}}{\partial x}+\frac{\partial^{2} w}{\partial x^{2}}\right)+A_{44} k_{S}\left(\frac{\partial \phi_{y}}{\partial y}+\frac{\partial^{2} w}{\partial y^{2}}\right)-N_{0} \frac{\partial^{2} w}{\partial x^{2}}=0
\end{aligned}
$$

Equations (8) are second-order differential equations with $\left(w, \phi_{x}, \phi_{y}\right)$ as the three unknowns.

\section{Finite element analysis}

The total potential energy $\Pi$ of a laminated plate under uniaxial loading is equal to strain energy $U$ can be written as [11-12]:

$\Pi=U_{b}+U_{s}+V$

where $U_{b}$ is bending term, $U_{s}$ is the strain energy of shear and $V$ denotes the potential energy of in-plane forces. The equilibrium requires that the potential energy $\Pi$ must be stationary. The equilibrium equations of the cross-ply laminated plate can be derived from the variational principle by using [11]

$\delta \Pi=0$

The finite element method is applied in this study, by using equations (8), (9) and (10) we obtain the following linear algebraic equations of the form:

$\left([K]+\lambda\left[K_{G}\right]\right)\{U\}=0$

where $[K]$ is the stiffness matrix, $\left[K_{G}\right]$ is the geometric stiffness matrix, $\lambda$ is the eigenvalue and $\{U\}$ is the nodal displacements. The critical buckling load $N_{x, c r}$ is that which corresponds to the least eigenvalue $\lambda$ determined by:

$$
\operatorname{Det}\left([K]-\lambda\left[K_{G}\right]\right)=0
$$

The non-dimensional buckling coefficient is given by $\mathrm{n}_{\mathrm{x} . \mathrm{cr}}$ is defined as $[3,7]$ :

$$
n_{x, c r}=\frac{N_{x, c r} b^{2}}{\pi^{2} \sqrt{D_{11} D_{22}}}
$$

where $N_{x, c r}$ is critical buckling load and $D_{11}$ and $D_{22}$ are bending stiffness [1]. 


\section{Materials and methods}

The critical buckling load for different plates depends on many parameters like as geometry of plate orientation of fiber layers and boundary conditions, have been computed by using ANSYS (Mechanical APDL 17.1); a finite element analysis program [12-13]. It should be noted that the use of this approach will frequently give an imprudent result compared to the real behavior of the structure (experimental).

In this study, the effects of circular cut-out on the buckling load of laminated composite plats have been investigated numerically. The mechanical properties of the E-Glass/Epoxy composite material are shown in Table 1. Perforated laminated plates were analysed for different boundary conditions: simple- simple- simplesimple (SSSS), simple- simple- simple-free (SSSF), simple-clamped- simple-free (SCSF), two different thickness $t=0.23 \mathrm{in} ; 0.355 \mathrm{in}$, and four width $b$ was varied from 6in, 5in, 4in and 3in and we kept diameter of hole constant $d=0.312 \mathrm{in}$. Meanwhile, the laminated plates were also analyzed without a hole to compare the influences having a hole and without a hole conditions on buckling loads [2]. Furthermore, the laminated plates were assumed to stack six laminas onto together namely cross-ply $[(90 \% /+\theta /-\theta)]_{\text {sym }}$ for symmetric and $[(90 \% /+\theta /-$ $\theta)]_{\mathrm{a}-\mathrm{sym}}$ for anti-symmetric ply with $\theta=15^{\circ}, 30^{\circ}, 45^{\circ}$ and $60^{\circ}$.

Table 1. Mechanical properties of the E-Glass/Epoxy.

\begin{tabular}{|c|c|}
\hline$E_{1}(\mathrm{Psi})$ & $5.49 \mathrm{E}+006$ \\
\hline$E_{2=} E_{3}$ (Psi) & $9.53 \mathrm{E}+005$ \\
\hline$G_{12}=G_{13}(\mathrm{Psi})$ & $3.47 \mathrm{E}+005$ \\
\hline$v_{12}=v_{13}$ & 0.3001 \\
\hline$v_{23}=v_{32}$ & 0.3001 \\
\hline
\end{tabular}

The SHELL 181 element "figure 2" is used for modeling and analyzing thin to moderately-thick perforated plates, it is a four-nodes element with six degrees of freedom at each node: translations in the $x, y$, and $z$ directions, and rotations about the $x, y$, and $z$-axes [13].

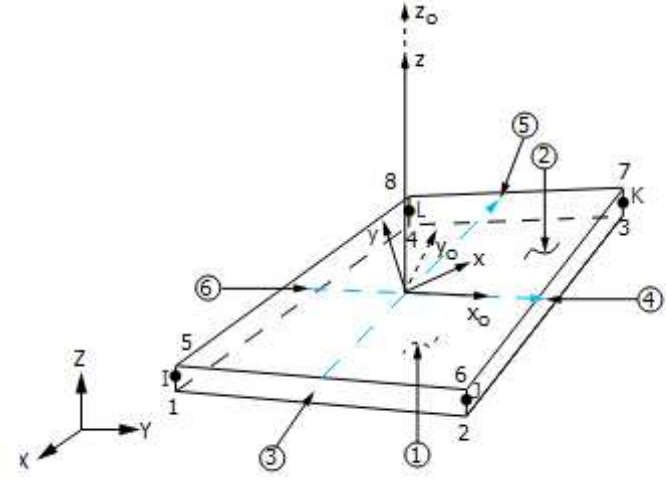

Fig. 2. Shell 181 element geometry [13].

\section{Numerical results and discussion}

Results obtained are shown in Tables 2, 3 and 4.

It is easily seemed from Tables that while thickness increase the critical buckling load $\mathrm{N}_{\mathrm{x}, \mathrm{cr}}$ increase.

"Figure 3" and "figure4" also refers that critical buckling of the boundary condition (SSSS) plates are approximately 2 times greater than the buckling load of (SSSF) and (SCSF) plates. The effects of $a / b$ ratio on buckling load are illustrated in "figure 5", according to it the buckling loads increase with increasing of $a / b$ ratios. Furthermore, the highest values of buckling loads are calculated for $\left[\left(90^{\circ} / 60^{\circ} /-60^{\circ}\right)\right]_{\text {sym }}$ (SSSS) plates, while the lower values are computed angle-ply for $\left[\left(90^{\circ} /+\theta /-\theta\right)\right]_{\text {sym }}$ (SSSF) plates. The effects of ply orientation symmetric and anti-symmetric on the buckling load can be seen in Tables 2, 3, 4. The results show that the change in buckling load are neglected. The effect of circular cut-out is taken into account. The analysis indicate that the variation of the buckling loads is very sensitive to the presence of hole. It can be seen that the buckling load generally decreases with presence of cut-out "figure 5", we can observe that the predicted buckling loads of plates without hole is $3.5 \%$ higher than with hole in (SSSS) plates and $22.5 \%$ in (SSSF) plates.

Table 2. Critical buckling load of perforated rectangular laminated plates under the boundary conditions (SSSS)

\begin{tabular}{|c|c|c|c|c|c|c|c|c|c|c|c|}
\hline \multirow{2}{*}{\multicolumn{4}{|c|}{ Plate dimensions (in) }} & \multicolumn{8}{|c|}{$\mathrm{N}_{\mathrm{x}, \mathrm{cr}}(\mathrm{kpsi})$} \\
\hline & & & & \multicolumn{4}{|c|}{ Symmetric $\left[\left(90^{\circ} /+\theta /-\theta\right)\right]_{\mathrm{sym}}$} & \multicolumn{4}{|c|}{ Anti-Symmetric $\left[\left(90^{\circ} /+\theta /-\theta\right)\right]_{\mathrm{a}-\mathrm{sym}}$} \\
\hline $\mathrm{a}$ & $\mathrm{b}$ & $\mathrm{t}$ & $\mathrm{d}$ & $15^{\circ}$ & $30^{\circ}$ & $45^{\circ}$ & $60^{\circ}$ & $15^{\circ}$ & $30^{\circ}$ & $45^{\circ}$ & $60^{\circ}$ \\
\hline \multirow{8}{*}{6} & 6 & \multirow{4}{*}{$\stackrel{\overbrace{}}{\tilde{o}}$} & \multirow{8}{*}{$\frac{n}{n}$} & 2.2556 & 2.4052 & 2.518 & 2.5361 & 2.2531 & 2.3886 & 2.528 & 2.5156 \\
\hline & 5 & & & 3.4902 & 3.663 & 3.8808 & 3.9489 & 3.4445 & 3.6124 & 3.8311 & 3.8747 \\
\hline & 4 & & & 5.5458 & 5.5539 & 5.671 & 5.7 & 5.5818 & 5.6488 & 5.7685 & 5.7159 \\
\hline & 3 & & & 9.4055 & 9.1028 & 9.4231 & 9.576 & 8.9998 & 9.1516 & 9.476 & 9.5089 \\
\hline & 6 & \multirow{4}{*}{$\begin{array}{l}n \\
n \\
n \\
0\end{array}$} & & 7.9823 & 8.4431 & 8.6962 & 8.8701 & 7.9824 & 8.4431 & 8.9297 & 8.8701 \\
\hline & 5 & & & 12.307 & 12.895 & 13.625 & 13.764 & 12.099 & 12.655 & 13.418 & 13.524 \\
\hline & 4 & & & 18.647 & 18.675 & 19.078 & 19.172 & 17.295 & 18.782 & 19.267 & 19.182 \\
\hline & 3 & & & 29.665 & 29.943 & 30.971 & 31.344 & 29.256 & 29.599 & 30.811 & 31.03 \\
\hline
\end{tabular}


Table 3. Critical buckling load of perforated rectangular laminated plates under the boundary conditions (SSSF)

\begin{tabular}{|c|c|c|c|c|c|c|c|c|c|c|c|}
\hline \multirow{2}{*}{\multicolumn{4}{|c|}{ Plate dimensions (in) }} & \multicolumn{8}{|c|}{$\mathrm{N}_{\mathrm{x}, \mathrm{cr}}(\mathrm{kpsi})$} \\
\hline & & & & \multicolumn{4}{|c|}{ Symmetric $\left[\left(90^{\circ} /+\theta /-\theta\right)\right]_{\mathrm{sym}}$} & \multicolumn{4}{|c|}{ Anti-Symmetric $\left[\left(90^{\circ} /+\theta /-\theta\right)\right]_{\mathrm{a}-\mathrm{sym}}$} \\
\hline a & $\mathrm{b}$ & $\mathrm{t}$ & $\mathrm{d}$ & $15^{\circ}$ & $30^{\circ}$ & $45^{\circ}$ & $60^{\circ}$ & $15^{\circ}$ & $30^{\circ}$ & $45^{\circ}$ & $60^{\circ}$ \\
\hline \multirow{8}{*}{6} & 6 & \multirow{4}{*}{$\stackrel{\overbrace{}}{0}$} & \multirow{8}{*}{$\frac{\mathfrak{m}}{3}$} & 1.0011 & 1.0114 & 1.0087 & 0.99357 & 1.0011 & 1.0184 & 1.0182 & 0.99804 \\
\hline & 5 & & & 1.0744 & 1.0977 & 1.0991 & 1.0741 & 1.0749 & 1.1103 & 1.1164 & 1.0822 \\
\hline & 4 & & & 1.1766 & 1.2454 & 1.2782 & 1.2435 & 1.1851 & 1.2695 & 1.3003 & 1.2466 \\
\hline & 3 & & & 1.4236 & 1.517 & 1.6469 & 1.5661 & 1.4435 & 1.6 & 1.6811 & 1.5776 \\
\hline & 6 & \multirow{4}{*}{$\begin{array}{l}n \\
\stackrel{n}{n} \\
0\end{array}$} & & 3.5699 & 3.61 & 3.5946 & 3.5269 & 3.565 & & 3.6288 & 3.5483 \\
\hline & 5 & & & 3.8231 & 3.9132 & 3.9136 & 3.8078 & 3.8211 & 3.9525 & 3.9721 & 3.8411 \\
\hline & 4 & & & 4.1833 & 4.432 & 4.5424 & 4.4041 & 4.2098 & 4.5121 & 4.6173 & 4.4183 \\
\hline & 3 & & & 5.0496 & 5.5714 & 5.8299 & 5.5265 & 5.1169 & 5.71 & 5.9475 & 5.5706 \\
\hline
\end{tabular}

Table 4. Critical buckling load of perforated rectangular laminated plates under the boundary conditions (SCSF)

\begin{tabular}{|c|c|c|c|c|c|c|c|c|c|c|c|}
\hline \multirow{2}{*}{\multicolumn{4}{|c|}{ Plate dimensions (in) }} & \multicolumn{8}{|c|}{$\mathrm{N}_{\mathrm{x}, \mathrm{cr}}(\mathrm{kpsi})$} \\
\hline & & & & \multicolumn{4}{|c|}{ Symmetric $\left[\left(90^{\circ} /+\theta /-\theta\right)\right]_{\mathrm{sym}}$} & \multicolumn{4}{|c|}{ Anti-Symmetric $\left[\left(90^{\circ} /+\theta /-\theta\right)\right]_{\text {a-sym }}$} \\
\hline a & $\mathrm{b}$ & $\mathrm{t}$ & $\mathrm{d}$ & $15^{\circ}$ & $30^{\circ}$ & $45^{\circ}$ & $60^{\circ}$ & $15^{\circ}$ & $30^{\circ}$ & $45^{\circ}$ & $60^{\circ}$ \\
\hline \multirow{8}{*}{6} & 6 & \multirow{4}{*}{ ֶి? } & \multirow{8}{*}{$\frac{c}{m}$} & 1.2129 & 1.2205 & 1.1927 & 1.1515 & 1.2104 & 1.2237 & 1.2001 & 1.1566 \\
\hline & 5 & & & 1.4313 & 1.4363 & 1.3817 & 1.3037 & 1.4291 & 1.4463 & 1.3975 & 1.3109 \\
\hline & 4 & & & 1.8848 & 1.9178 & 1.8306 & 1.6693 & 1.8836 & 1.9302 & 1.8416 & 1.6664 \\
\hline & 3 & & & 3.2651 & 3.3557 & 3.1656 & 2.7395 & 3.2662 & 3.3677 & 3.1688 & 2.7342 \\
\hline & 6 & \multirow{4}{*}{ ڤn } & & 4.3048 & 4.3374 & 4.2351 & 4.0777 & 4.2899 & 4.3429 & 4.2612 & 4.102 \\
\hline & 5 & & & 5.0578 & 5.0874 & 4.8951 & 4.6072 & 5.0428 & 5.1132 & 4.9466 & 4.6382 \\
\hline & 4 & & & 6.635 & 6.7591 & 6.4548 & 5.8859 & 6.6209 & 6.7905 & 6.4898 & 5.881 \\
\hline & 3 & & & 11.351 & 11.653 & 11.02 & 9.5718 & 11.344 & 11.689 & 11.033 & 9.56 \\
\hline
\end{tabular}
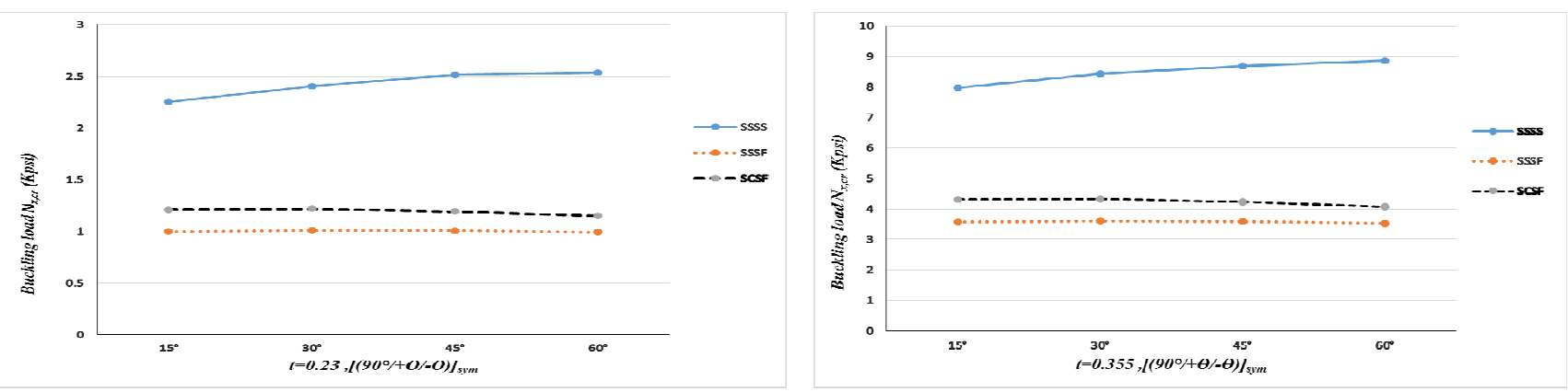

Fig. 3. Variation of the critical buckling load $N_{x, \text { cr }}$ compared to symmetric orientation of fibers of a plate $6 \times 6$ in $^{2}$ for $t=0.23$ in and $\mathrm{t}=0.355$ in
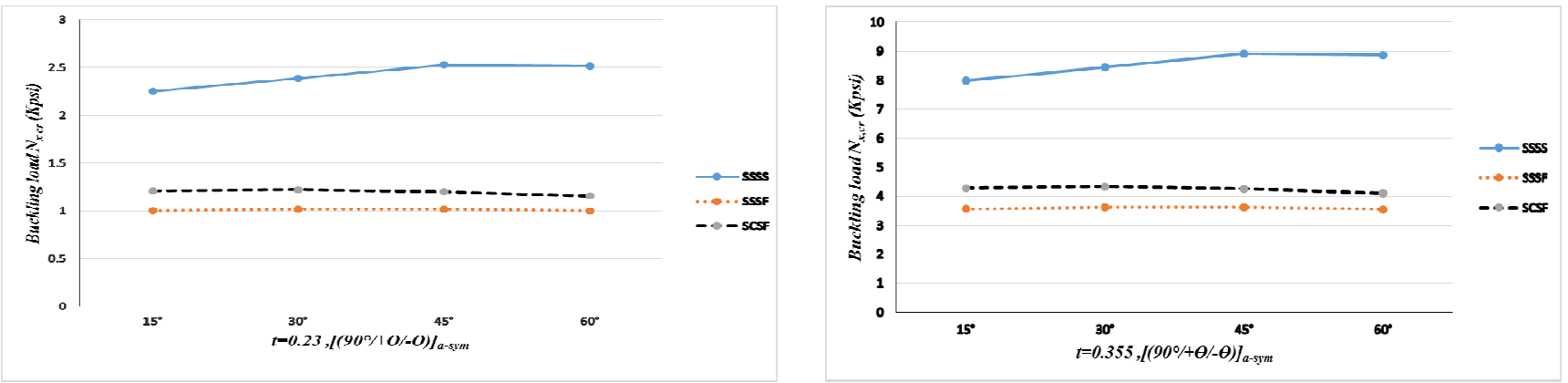

Fig. 4. Variation of the critical buckling load $\mathrm{N}_{\mathrm{x}, \mathrm{cr}}$ compared to anti-symmetric orientation of fibers of a plate $6 \times 6$ in ${ }^{2}$ for $t=0.23$ in and $\mathrm{t}=0.355$ in 

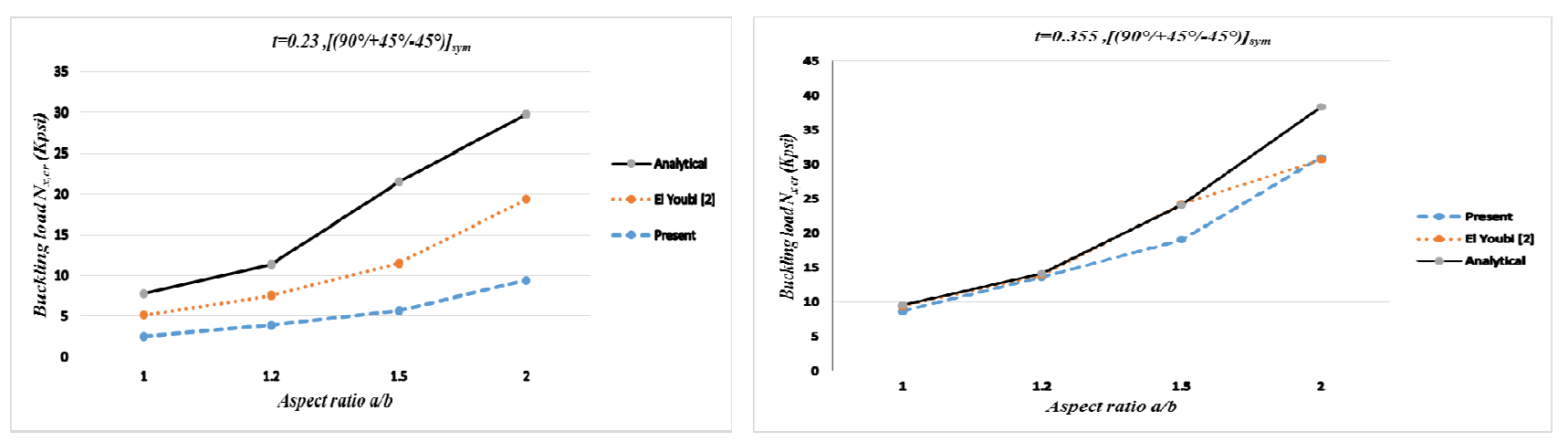

Fig. 5. Variation of the critical buckling load $\mathrm{N}_{\mathrm{x}, \mathrm{cr}}$ compared to aspect ratio $\frac{a}{b}$ for $\left[\left(90^{\circ} / 45^{\circ} /-45^{\circ}\right)\right]_{\mathrm{sym}}[2]$

\section{Conclusion}

In this study, the buckling load of a E-Glass/Epoxy composite laminated rectangular plate with central circular cut-out is investigated. Additionally, the effect of $\mathrm{a} / \mathrm{b}$ ratio on buckling loads are calculated. Solutions were done with FEM using ANSYS finite element program. From the present study, the following conclusions can be made:

1. The reduction of the buckling load due to hole is found to be significant, this means big holes cause the weakest plates under the pressure.

2. The increasing of buckling loads by increasing $a / b$ ratios.

3. Boundary conditions of composite have the strongest effect on the buckling load, among the other parameters.

4. Buckling is loss of stability due to geometric effects and boundary conditions than the material failure.

Comparison of results of the finite element with test have to be done.

\section{References}

1. J.M. Berthelot, Composite Materials: Mechanical Behavior and Structural Analysis (Springer-Verlag, New York, 1999)

2. M. El Youbi, M. Rougui, Int. Rev. Mech. Eng. 9, 3 p.307-313 (2015)

3. M.P. Nemeth, J. Ther. Str. 23, 9 p.873-916 (2000)

4. B. O. Baba, J. of Reinf. Plast. and Comp. 26,16 p. 1637-1655 (2007)

5. S. Vlachoutsis, Int. J. for Num. Meth. in Eng. 33, p.1537-1552 (1992)

6. S.N. Chatterjee, S.V. Kulkarrni, A. I. A. A. J. 17, 5, p.498-499 (1997)

7. M.F. Altan, M.E. Kartal 30, p.2243-2249 (2009)

8. W. Kim, J.N. Reddy, L. Amr. J. Sol. Str. 7, p.201$226(2010)$

9. B.D. Agarwal, L.J. Broutman, K. Chandrashekhara Analysis and Performance of Fiber Composites (Wiley, USA, 2006)
10. J.N. Reddy, Mechanics of Laminated Composite Plates and Shells : Theory and Analysis (CRC Press, Boca Raton, 2004)

11. J.N. Reddy, An Introduction to Nonlinear Finite Element Analysis (Oxford University Press, New York, 2004)

12. E. Madenci, I. Guven, The finite element method and applications in Engineering using ANSYS (Springer, New York, 2015)

13. ANSYS Mechanical APDL Theory reference, Release 17.1 